\title{
Breakfast of Champions: Fast Lipid Accumulation by Cultures of Chlorella and Scenedesmus induced by Xylose
}

\author{
Gustavo B. Leite ${ }^{1}$, Kiran Paranjape ${ }^{1}$, and Patrick C. Hallenbeck ${ }^{1,2 *}$ \\ ${ }^{1}$ Département de microbiologie, infectiologie, et immunologie, Université de \\ Montréal, \\ CP 6128, Centre-ville, Montréal. PQ H3C 3J7 Canada \\ ${ }^{2}$ Life Sciences Research Center, Department of Biology \\ United States Air Force Academy \\ 2355 Faculty Drive, USAF Academy, Colorado 80840
}

*Corresponding author: patrick.hallenbeck@umontreal.ca, fax: 514-343-5701

\begin{abstract}
The use of wastewater for algal cultivation could couple waste treatment credits with access to water and low-cost nutrients. The pulp \& paper industry produces a waste stream rich in hemicellulose, where the major component is xylose. This pentose was previously shown to be relatively toxic to algal cells. Here its short terms effects on Chlorella sp. and Scenedesmus sp. strains were evaluated. Xylose induced a quick boost in the cellular lipid content of the Chlorella strains, with flow cytometry data showing an increase of up to 4.1 fold in only six to twelve hours, accompanied by growth arrest. After peak lipid accumulation, these strains started losing chlorophyll with complete loss over the next few days. However, addition of xylose to cultures of the Scenedesmus $s p$. did not show any negative effects. This strain was shown to be capable of both mixotrophic growth in the light and dark heterotrophic growth with xylose. These results


suggest that xylose could be a substitute for the nitrogen starvation strategy for lipid content enhancement in Chlorella sp. strains, with higher performance in a fraction of the time required by the nutrient starvation method; and as carbon source for Scenedesmaceae strains.

\section{Keywords}

Microalgae, Chlorella vulgaris, Xylose, Lipid Enhancement, Mixotrophic Cultivation, Algal Biofuels 


\section{Introduction}

Algal biodiesel is a third generation biofuel with potential for sustainable fuel production with a small footprint and reduced competition with food crops [1] . Although algal cultivation does not require arable land, it has a large demand for water, fertilizers and solar radiation, which drastically limits suitable locations [2]. A series of strategies are being developed to circumvent these issues, such as; the use of marine species [3], cultivation coupled with wastewater treatment plants [4-6], and mixotrophic cultivation using cheaply available or industrial waste derived carbon sources [7,8]. Different carbon sources have been evaluated for mixotrophic or heterotrophic microalgal cultivation, including, glucose [9], acetate [10], glycerol [11] and domestic wastewater [7]. The use of xylose (monomer) or xylan (polymer) has recently been reported for microalgal cultivation, either under mixotrophic [12,13] or heterotrophic conditions [14], or in a consortium with bacteria specialized in conversion of this pentose [15].

Xylose is among the major components of hemicellulose and thus an abundant carbon source [16]. Hemicellulose, present in plant cell walls, has an amorphous and less rigid structure, in comparison with cellulose. It is a heteropolymer formed by different sugars in various proportions depending upon the plant [16]. Corn straw, for example, is rich in xylan and represents a good source of this pentose [17]. Xylose is also a significant component of black liquor, a waste product produced by the pulp and paper industry that is presently burned after energy intensive concentration processes. There are a number of challenges to using this sugar for second-generation biofuels, primarily bioethanol. Chief among these is the inability to effectively use the hemicellulose fraction of lignocellulose due either to xylose toxicity or the general inability of industrial strains 
to ferment both glucose and xylose [18-20]. One study reported that the toxicity of the xylose is associated with the inactivation of hexokinases, inhibiting the first step of glycolysis [20]. Another issue found in the yeast Saccharomyces cerevisiae is related to the transmembrane transport of xylose. It is pumped into the cell by glucose transporters, but low affinity for xylose compromises its assimilation when glucose is available [21].

The first report of algal cultivation with xylose showed that some strains were tolerant and presumably assimilated it through mixotrophic growth, with however serious toxicity in some strains [13]. The second report showed that some strains could indeed assimilate xylose under mixotrophic or heterotrophic cultivation conditions, with different taxonomic groups showing different tolerances and assimilation capabilities [14]. Recently, a report showed the successful use of xylose by a consortium, with a bacterium, Cellvibrio pealriver, transforming xylose into assimilable organic acids that are then assimilated by an alga [15]. Zheng et al. showed that assimilation of xylose by a member of the Trebouxiophyceae was greatly increased when its glucose transporters had been previously activated [12].

Thus, it is clear that some algae assimilate xylose, but that this capability varies among species, with potential toxic effects observed in some cases. However, no information is available about the physiological effects that xylose might induce in an exponentially growing algal culture, or its potential impact on biomass and lipid production. In these previous reports on mixotrophic and heterotrophic algal cultivation using xylose as alternative carbon source, cultures were exposed to xylose throughout growth $[13,14]$. These studies reported long lag phases and substantial pigment loss by the algal cells, suggesting that the cells were under significant physiological stress. 
We are interested in mixotrophic growth for microalgal cultivation as supplementation with a cheap and readily available carbon source may provide a strategy to boost algal biofuel production in cold climate regions. As a first step in optimization of cultivation yields, the present work aimed to analyze in detail the short term and longer term effects of xylose addition to algal cultures. Flow cytometry can monitor cell size and the cellular content of neutral lipids, chlorophyll and accessory pigments, potentially giving information about stress and different cellular adaptations over the short term. Here flow cytometry was used to analyze the short-term effects caused by xylose addition on biomass, lipid and chlorophyll content in growing algal cultures, which showed a marked boost in lipid accumulation over a very short initial period ( $6 \mathrm{~h}$ to $12 \mathrm{~h}$ ). Thus, these results suggest a different mixotrophic cultivation strategy, exposing cultures to xylose only after a previous photoautotrophic growth period, saving on feedstock costs and increasing productivity. Thus, the use of xylose might increase the feasibility of algal biofuel production in cold climates.

\section{Methods}

\subsection{Strains and Culture Conditions}

To analyze the physiological impact that xylose might have on algal cells, four different strains of microalgae native to Québec were chosen [22]. Bold's Basal Medium (BBM), [23], modified by the addition of $50 \mathrm{mM}$ MES pH 7.4, was used throughout. The pre-inocula were grown under photoautotrophic conditions: $125 \mathrm{~mL}$ Erlenmeyer flasks containing $50 \mathrm{~mL}$ of medium, with agitation $(100 \mathrm{rpm})$ and illuminated in a light-dark 
cycle of 12 hours with warm white fluorescent light bulbs at an intensity of $35 \mathrm{~W} / \mathrm{m}^{2}$ (approximately $166 \mu \mathrm{E} / \mathrm{m}^{2} / \mathrm{s}$ ), measured with the photo-radiometer Delta OHM HD2102.1, equipped with the probe LP 471 RAD. Pre-inocula were grown until mid-log phase, diluted with medium to an optical density of 0.5 at $630 \mathrm{~nm}\left(\mathrm{OD}_{630}\right)$ and used as inocula $(10 \%(\mathrm{v} / \mathrm{v}))$.

The experiments consisted of two stages, differentiated by the time of addition of xylose. Thus, the time points in this study make reference to the day when xylose was added to the cultures. In the first stage, cultures were initially allowed to grow for three days before samples were collected for data acquisition. Cultures were then followed for two days (first stage) (data points: $t=-48 \mathrm{~h}$ and $\mathrm{t}=-24 \mathrm{~h}$ ), after which xylose was added $(t=0 h)$ to a final concentration of $30 \mathrm{mM}$. In the second stage $(t>0 h)$, the cultures of each strain were divided into four and incubated under four distinct conditions: (i) mixotrophic, where the cultures were kept under the same light conditions as the first stage, but xylose (BioShop) was added to a final concentration of $30 \mathrm{mM}$ from a master solution of 500mM; (ii) heterotrophic, where the cultures were supplemented with xylose to $30 \mathrm{mM}$, but switched to dark incubation; (iii) photoautotrophic, where the cultures were kept under the same conditions as the first stage; (iv) dark autotrophic, where the culture from the first stage was simply switched to dark incubation. These cultures were analyzed over several time-points during stage one and two (as indicated) to reveal short and long term physiological changes as inferred from alterations in cell size, chlorophyll content, lipid content, optical density and culture $\mathrm{pH}$. 


\subsection{Flow Cytometry Analysis}

Using a BD FACSort Flow Cytometer, multi-parameter analyses were performed to reveal shifts in cell metabolism and fitness. This instrument uses a laser of $488 \mathrm{~nm}$ excitation; three emission detectors: FL1 (515-545nm); FL2 (562-607nm) and FL3 (>630nm); a forward scatter detector (FSC); and a side scatter detector (SSC). Data acquisition was gated above the noise threshold and bacterial interference, allowing only readings of algal cells. Forward (FSC channel) and side light scattering (SSC channel), mean fluorescence of the FL1 channel, the fluorescence output of BODIPY 505/515® (neutral lipid dye, see below for details), and FL3 channel, the natural chlorophyll autofluorescence, were recorded at different time points over a 6 day period.

Samples were stained with BODIPY® 505/515 (4,4-difluoro-1,3,5,7-tetramethyl4-bora-3a,4adiaza-s-indacene (ThermoFisher Scientific) to a final concentration of $0.5 \mu \mathrm{M}$ and 5\% DMSO (Bio Shop), as described elsewhere [24, 25], to follow changes in cellular lipid content (relative fluorescence) by flow cytometry (FL1). The observed relative fluorescence intensities were related to neutral lipid content as describe below in section 2.3. BODIPY® $505 / 515$ is a lipophilic dye that emits a strong green fluorescence. For flow cytometry analysis of algal lipids, this dye has the advantage of emitting fluorescence at a wavelength that is very distinct from that of chlorophyll. It is read through the FL1 channel, while the chlorophyll is detected on the FL3 channel.

\subsection{Lipid and Biomass Quantification}

BODIPY $^{\circledR} 505 / 515$ is widely used to visualize neutral lipids of microalgal cells. Flow cytometric analysis of cells stained with this fluorescent dye, read via the FL1 channel, 
gives a fluorescent density distribution in arbitrary fluorescence units (A.U.). However, it is not very useful for microtiter plate analysis due to its high background signal. Thus, it is necessary to make a correlation between these values and an independent measurement in order to make the FL1 channel results quantitative. Nile Red was used to establish a correlation between lipid content and FL1 (BODIPY ${ }^{\circledR}$ 505/515) output. Nile red is an efficient dye for quantification of neutral lipids [26-28] and unlike BODIPY $^{\circledR} 505 / 515$, the fluorescence emission wavelength of Nile Red is solvent dependent. Thus, Nile Red does not emit fluorescence in water, a useful property for lipid quantification in multiwell plates. Calibrated with the proper standards, this method gives the absolute quantity of lipid in a fixed volume of algal culture with known biomass concentration.

Here, a Nile Red assay was used to determine cellular lipid content as percent of lipid per biomass dry weight and to then correlate this with the mean cellular lipid content found through flow cytometry. The procedures described elsewhere [14] were used with slight modifications. 50 $\mathrm{L}$ of algal culture were incubated with Nile Red (Sigma) (final concentration $0.5 \mu \mathrm{g} / \mathrm{mL}$ ), and dimethylsulfoxide (DMSO) (25\%) for fifteen minutes with agitation using a microtiter plate shaker (DSG Titertek Flow Laboratories, Meckenheim, Germany). The plates were read using a Synergy NEO HTS Microplate Reader set for excitation at $520 \mathrm{~nm}$ and emission detection at 570nm. Fluorescence output was correlated to lipid concentration through a standard curve $\left(\mathrm{R}^{2}=0.9919\right)$ made with known quantities of olive oil $\left(\operatorname{Lipid}_{(\mathrm{mg} / \mathrm{L})} /\right.$ Fluor.(A.U.) $\left.=0.0092166\right)$. The standard curve was developed using triplicate samples in the same 96 well plate as the samples. The lipid content of the algal biomass as percent of dry weight was then calculated by dividing the determined lipid concentration $(\mathrm{mg} / \mathrm{L})$ by the corresponding biomass concentration $(\mathrm{mg} / \mathrm{L})$. The lipid 
content found using Nile Red was then correlated with the output of the flow cytometer FL1 channel, using the same algal sample, this time stained with BODIPY 505/515. This

gave a linear $\left(\mathrm{R}^{2}=0.93207\right)$ correlation between FL1 output and lipid content $\left(\operatorname{Lipid}_{(\% \mathrm{dw})} /\right.$ FL1 output $_{(\text {A.U. }}=0.00243$ ). All the lipid data presented are the average of three analytical readings (technical replicates) of biological triplicates (independent experiments).

To develop a standard curve correlating algal biomass dry weight with the corresponding optical density at $630 \mathrm{~nm}$, algal cultures with different biomass concentrations were analyzed in a Shimadzu UV/VIS 2100PC spectrophotometer to determine the optical density at $630 \mathrm{~nm}(\mathrm{OD} 630 \mathrm{~nm})$, transferred to pre-weighed $1.5 \mathrm{~mL}$ centrifuge tubes, centrifuged at 16000g for 5 minutes (Sigma Laborzentrifugen D-37520), the supernatant removed and the tubes placed in an oven at $110^{\circ} \mathrm{C}$. The tubes were then weighed again and the biomass was taken as dried when the weight measured over time had stabilized. The biomass weight obtained by subtracting the weight of the empty tube was correlated with the corresponding optical density values to build a standard curve $(\mathrm{R} 2=0.8936)$ and the correlation factor (Biomassg/L /OD630nm=0.9758) was used to obtain the algal biomass dry weight from optical density readings.

\subsection{Spectrophotometric Determination of Xylose}

For xylose quantification, a method previously described in small volume samples [14] was used with slight modifications. This method is a scaled down version of a

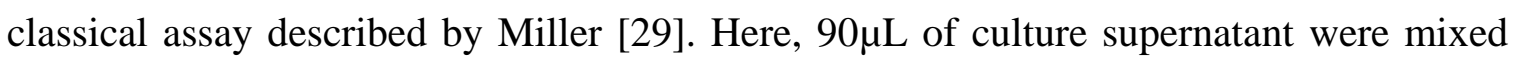
and incubated for 15 minutes in a water bath at $90^{\circ} \mathrm{C}$ with $90 \mu \mathrm{L}$ of a DNS solution $(10 \mathrm{~g} / \mathrm{L}$ 3,5-dinitrosalicylic acid (Sigma Aldrich); 10g/L sodium hydroxide (Bio Shop); 0.5g/L 
sodium sulphate (Bio Shop)). After incubation, the 96 well plates were cooled down immediately using an ice bath and the color development stabilized by the addition of $30 \mu \mathrm{L}$ of $40 \%$ potassium sodium tartrate solution (Fisher). The optical density at $515 \mathrm{~nm}$ was recorded using a Biotek EL800 microtiter plate reader and compared to a standard curve made up in the same multi-well plate.

\subsection{Molecular Identification}

A preliminary identification of the chosen strains as three distinct Chlorella and one Scenedesmus was previously made using optical microscopy [14]. To confirm this, analyses were performed using DNA sequences of an internal region of the gene for $18 \mathrm{~S}$ ribosomal RNA: (i) samples were compared to the NCBI database for simple alignment comparison; (ii) the DNA sequences were aligned with known strains from established culture collections, and similarities and divergences were expressed in the form of a tree of life. The targeted DNA was amplified and sequenced using the primers 18S-intforward 5'-GTGGTAACGGGTGACGG-3' and 18S-int-reverse $\quad$ 5' $^{\prime}$ GTGCGGCCCAGAACATC-3' (Designed using CLC Main Workbench (version 6.8.4) and synthesized by Alpha DNA). PCR products were purified and sequenced using the Sanger method at IRIC Genomics Platform (https://genomique.iric.ca). Chromatographic data were analyzed with CLC Main Workbench (version 6.8.4) software, and the DNA sequences obtained were deposited in GenBank (NCBI) under the accession numbers KT587785 (LB1H09), KT587786 (LB1H10), KT587787 (PCH03) and KT587788 (PCH90). The phylogenetic tree was made using MEGA (version 5.2.2) software, through the neighbor joining method and a Jukes-Cantor model at a bootstrap of 1000 . 


\subsection{High-Performance Liquid Chromatography (HPLC) Analysis for Organic Acids}

Established HPLC methods [30, 31] were used to detect the presence of any organic acids that could have been secreted into the medium. The supernatant of the cultures at different time points $(-12 \mathrm{~h}, 0 \mathrm{~h},+6 \mathrm{~h},+12 \mathrm{~h},+24 \mathrm{~h},+48 \mathrm{~h})$ were examined using a Prevail Organic Acid Column, 150x4.6mm 5 $\mathrm{m}$ (Grace Davison Discovery Sciences) and a Beckman-Coulter HPLC Gold system equipped with a 126 solvent module and a 168 UV spectrophotometric detector. The column was developed with a mobile phase consisting of $25 \mathrm{mM} \mathrm{KH}_{2} \mathrm{PO}_{4}$ adjusted to $\mathrm{pH} 2.5$ with phosphoric acid. The flow rate was set to $1 \mathrm{ml} / \mathrm{min}$, the column was at room temperature $\left(20^{\circ} \mathrm{C}\right)$, and detection was at $210 \mathrm{~nm}$. At each time point, a sample of $1 \mathrm{~mL}$ of the culture was centrifuged, the supernatant collected and filtered using a $0.22 \mu \mathrm{m}$ membrane filter.

\subsection{Statistical Analyses}

Statistical analyses were made using Prism 6.0D (GraphPad) software. To determine the statistical significance between the photoautotrophic, autortrophic, mixotrophic and heterotrophic groups, the two-tailed t-test, with the confidence interval of $95 \%$ or two-way ANOVA were used according to their intrinsic suitability. 


\section{Results and Discussion}

\subsection{Strain Identification}

To identify the strains used in this work, an internal portion of the gene coding for 18S rRNA (912bp) was amplified, sequenced, compared against the NCBI database, aligned and compared to other known species for their placement in the proper phylogenetic clade. A comparison of the sequences obtained with the ones present in the NCBI database revealed that three of the strains (PCH03, PCH90, LB1H09) were within the Chlorella genus, with a fourth strain (LB1H10) belonging to the family Scenedesmaceae. Placing these strains into taxonomic groups requires the construction of a phylogenetic tree. This is based on the alignment of DNA sequences of 18S rRNA of established culture collection strains (Figure 1). This tree, the output of the MEGA program, is drawn to scale, with branch lengths reflecting the evolutionary distances between organisms. The tree generated with the culture collection strains and the wildtype strains of the present study was rooted only at the phylum level (Chlorophyta), revealing the distance between $\mathrm{LB} 1 \mathrm{H} 10$ (Chlorophyceae) and the other three strains in this study, associated with the class Trebouxiophyceae. Four different species of the genus Chara were used to root the four strains in this study under the kingdom Plantae. The genus Chara is organized under the phylum Charophyta, one clade above the divergence of LB1H10 and the other strains. The Chlamydomonadales order served as an outgroup for LB1H10, grouping it inside the Scenedesmaceae family. This strain showed a high similarity to the genera Scenedesmus and Acutodesmus, but there was not enough definition to distinguish which one was the most related (Figure 1). The three other 
strains: LB1H09, PCH03, PCH90 were classified as members of the genus Chlorella. The genera Prasiola and Lobosphaera differ from Chlorella at the order level (orders Prasiolales and Trebouxiales respectively). The genus Micractinum is related to Chlorella vulgaris at the family level and was used as to root the genus, while the culture collection typed strain Chlorella sorokiniana was used to root LB1H09, PCH03 and PCH90 as different strains of Chlorella vulgaris. The level of similarity was too high to resolve the exact positions of the strains $\mathrm{PCH} 03$ and $\mathrm{PCH} 90$ with respect to A1-65 (accession number KF661335.1), CCAP 211/21A (accession number KJ756823.1) and D2 (accession number JX185298.1). The strain LB1H09, although also a strain of Chlorella vulgaris, was consistently separated from the others (Figure 1).

\subsection{Effects of xylose addition on growth and cell size}

The strains used in this work had previously been shown to be capable of heterotrophic growth using xylose as a carbon source [14]. Here, we examined their performance under a two-stage cultivation regime and characterized the short-term effects of the addition of xylose as well as the time required for metabolic adaptation. After the first stage photoautotrophic cultivation, the cultures were divided and incubated under four different conditions: photoautotrophic, mixotrophic, heterotrophic (dark) and dark autotrophic. As might be expected, cultures that continued to be incubated under photoautotrophic conditions grew over the rest of the experiment ( $96 \mathrm{~h}$ ) and, cultures incubated under autotrophic conditions in the dark ceased immediately to grow with a decline in OD over the rest of the experiment (up to 96h) (Figure 2, left hand panels). Addition of xylose and incubation in the dark allowed all the cultures, after an initial lag

phase, to grow heterotrophically over the rest of the experiment (Figure 2, left hand 
panels). Different strains reacted differently in the long term to mixotrophic conditions, with the addition of xylose allowing greater growth in the light than photoautotrophic conditions for strain LB1H10, but less growth, i.e. apparent inhibition, with strains LB1H09, PCH03 and PCH90 (Figure 2, left hand panels).

Flow cytometry can also be used to follow apparent relative changes in cell size with time by analysis of forward scatter (FSC). The size of algal cells is usually found within a specific range during growth, but can shift depending on physiological state and species [33-35]. Strain LB1H10, a member of the Scenedesmaceae family, can be found as solitary cells or as coenobium of two to four cells, exhibited an anomalous FSC pattern that can be interpreted as being due to the formation of coenobium over the first $48 \mathrm{~h}$ (from $-48 \mathrm{~h}$ to $0 \mathrm{~h}$ ). This confounds further analysis so in what follows the results with this strain will not be presented. As expected, since all of them belong to the same genus, strains LB1H09, PCH03, and PCH90 presented similar FSC patterns throughout the experiment. When FSC is examined with the cultures of LB1H09, PCH03 and PCH90, two effects can be seen. As expected, photoautotrophically grown cultures retained the same inferred cell size over time as they had at $\mathrm{t}=0$. However, placing cultures in the dark induced for all cultures a decrease in relative cell size over time (Figure 2, right hand panels). Likewise, addition of xylose, caused an overall decrease in cell size independently of whether the cultures were incubated in the light or the dark (Figure 2, right panels).

Although the above effects were noted over the length of the experiment, significant effects were also seen in the second stage in the very short term, as seen by the growth rate determined over the first 24 hours after the addition of xylose (Table 1). All strains 
incubated in the light with xylose (mixotrophic conditions) gave a slower growth rate when compared to photoautotrophic conditions, and the growth of two strains, LB1H09 and $\mathrm{PCH} 03$, virtually halted during this period. All strains incubated under heterotrophic or dark autotrophic conditions showed a significant short term biomass loss, which was slower in the presence of xylose (heterotrophic conditions) than in its absence (dark autotrophic) (Table 1). This loss is in agreement with a recent report on biomass loss by microalgae during the dark period of light/dark regime [32]. After this initial period, all strains incubated under heterotrophic conditions resumed growth, three after a lag phase of twelve hours (PCH03, PCH90, and LB1H10), and one after a twenty-four hour lag phase (LB1H09) (Figure 2, left hand panels).

The addition of xylose to some of the strains examined brought about striking visual changes regardless of whether or not the cultures were subsequently exposed to light (Figure 3). Only two days were sufficient to see a significant bleaching effect due to the loss of chlorophyll, and all of three Chlorella vulgaris strains tested (PCH03, PCH90 and LB1H09) appeared almost devoid of chlorophyll by the fourth day (Figure 3, left panels). The only exception was the strain belonging to the Scenedesmaceae family, LB1H10, which maintained a healthy appearance under both mixotrophic and photoautotrophic conditions (Figure 3). This effect seemed to be specific to the presence of xylose and not to the depletion of some nutrient, e.g. nitrate, since cultures incubated for the same period of time in the light in the absence of xylose apparently maintained their chlorophyll levels (Figure 3, right panels).

The $\mathrm{pH}$ of the cultures incubated under mixotrophic and photoautotrophic conditions was followed in order to determine whether any of the observed effects could 
be caused by $\mathrm{pH}$ stress. Despite the use of 50mM MES buffer, the $\mathrm{pH}$ of these cultures varied depending upon strain and treatment (Figure 4). As expected, the $\mathrm{pH}$ of all the cultures under photoautotrophic conditions increased over time, and at the end of the experiment was essentially the same for all strains $(\mathrm{pH}=10.93 \pm 0.15)$. The addition of xylose to cultures of three of the strains induced an acidification whose level and timing varied for each strain (Figure 4). The pH of mixotrophic cultures of LB1H09 and PCH03 started dropping appreciably after 48 hours, reaching a final $\mathrm{pH}$ of 5 at 96 hours. On the other hand, strain PCH90 reacted differently to xylose addition as it maintained a relatively stable $\mathrm{pH}$ under mixotrophic conditions $(\mathrm{pH}=7.74+0.07)$. As well, the $\mathrm{pH}$ of a mixotrophic culture of LB1H10 dropped near the end of the experimental period, after $72 \mathrm{~h}$, but since it had risen prior to this, the $\mathrm{pH}$ of this culture at the end of the incubation was still near neutrality. Analysis for organic acids using HPLC did not reveal any differences before or after the addition of xylose in the cell-free supernatants of any of the mixotrophic cultures, suggesting that the observed acidification was not a result of organic acids being released into the medium.

Taken together, these results suggest that the chlorophyll loss evident in cultures with xylose is: (i) species specific; (ii) not due to acidification of the medium, since the medium $\mathrm{pH}$ of strains $\mathrm{PCH} 90$ and $\mathrm{PCH} 03$ remained in the neutral zone for the first 72 hours after the addition of xylose; (iii) not likely due to photo-oxidative stress, as the cultures kept in the dark showed similar or worse chlorophyll loss than cultures incubated in the dark in the absence of xylose (see section 3.3 for details). 


\subsection{Effects of Xylose Addition on Chlorophyll Fluorescence and Cellular Lipid Content}

The chlorophyll loss noted qualitatively above (Section 3.2 and Figure 3 ) is in agreement with a previous report of the mixotrophic and heterotrophic utilization of xylose by microalgae which noted that cultures were pale white when this sugar was present [13]. To examine this in more detail, we used flow cytometry to analyze quantitatively chlorophyll fluorescence before and after the addition of xylose. The FL3 channel of the flow cytometer used is equipped with a $630 \mathrm{~nm}$ long pass filter (LP), allowing the analysis of chlorophyll fluorescence without interference by BODIPY ${ }^{\circledR}$ fluorescent dye, and thus providing reliable information on cellular chlorophyll content.

Flow cytometric analysis of all culture conditions started at $\mathrm{t}=-24 \mathrm{~h}$. During the next 24h (until 0h), the amount of chlorophyll per cell was stabile (Figure 5). As expected, after time point $\mathrm{t}=0 \mathrm{~h}$, all strains shifted to dark autotrophic conditions sustained a slow decrease in chlorophyll content. In contrast, when xylose was added and the cultures were incubated in the dark (heterotrophic conditions), all strains showed a strong decline in chlorophyll content, reaching the lowest levels observed (Figure 5, right panels). The difference in the decrease of chlorophyll fluorescence between the dark autotrophic and heterotrophic conditions was statistically significant $(\mathrm{P}<0.05)$ for three of the four strains, the exception was $\mathrm{PCH} 90(\mathrm{P}=0.1171)$. This exception agrees with the results described in the previous section and elsewhere [14], suggesting that this strain is not as efficient as the other strains in using xylose to support mixotrophic or heterotrophic growth. 
The photoautotrophic cultures were rather stable in terms of chlorophyll fluorescence (Figure 5, right panels). The small decrease seen between time points $12 \mathrm{~h}$ and $24 \mathrm{~h}$ is likely due to the periodicity of the light/dark regime, as this coincided with the dark period of the cycle. As for the cultures under mixotrophic conditions, the addition of xylose quickly affected the levels of chlorophyll, with fluorescence fading significantly over time similarly for three of the strains (PCH03, PCH90, and LB1H09) tested ( $<$ 0.01) (Figure 5, right panels). The exception was strain LB1H10, which despite the variation seen over the different time points and within replicates (Figure 5, right panels), presented relatively stable chlorophyll content, as evidenced by mean FL3 channel fluorescence. For LB1H10, the cellular chlorophyll content under mixotrophic conditions was comparable to that observed under photoautotrophic conditions. Since the three strains showing a decrease in chlorophyll content (all C. vulgaris) under mixotrophic conditions also presented the same pattern when cultivated heterotrophically in the dark, these results show that the influence of xylose on cellular chlorophyll content is not light dependent.

The fluorescent dye BODIPY ${ }^{\circledR} 505 / 515$ can be used in flow cytometry to visualize the lipid content of algal cells in a sensitive and reliable manner [24,33]. The wavelength of emitted fluorescence is far from natural chlorophyll autofluorescence. In addition, this dye easily penetrates cells, allowing for the analysis of live cells and making this method robust [34]. Here we used flow-cytometry of BODIPY ${ }^{\circledR}$ stained cells to analyze the short-term effects of xylose addition. The results reveal a large rapid increase in the cellular lipid content of mixotrophic cultures, but only a slight increase in the cellular lipid content of heterotrophic cultures (Figure 5, left panels). Two of the 
strains under mixotrophic cultivation, strains LB1H09 and PCH90, increased their lipid content 2.9 and 4.1 fold in 12 hours respectively, while strain PCH03 gave a 2.7 fold increase in only 6 hours. The increase in cellular lipid content does not seem to be linked to any change in cell morphology or size, as ascertained using channels SSC and FSC; disassembly of the photosynthetic apparatus, determined using channel FL3 (chlorophyll); or $\mathrm{pH}$ stress. This suggests that the observed xylose toxicity might be related to metabolite accumulation (e.g. xylitol, d-xylulose) or some type of regulatory interference with metabolism. In mammals, a metabolite, xylulose-5-phosphate, which is also a putative intermediate in the algal xylose assimilation pathway, is directly involved in the regulation of lipogenesis genes [35].

\subsection{The Effect of Xylose on Total Biomass and Total Lipid Production}

To better characterize the effects of xylose addition, its consumption and the total increases in biomass and volumetric lipid production were quantitated. All the strains examined showed some degree of xylose consumption under mixotrophic growth conditions (Table 2). Xylose was added to the algal cultures to a final concentration of $4.5 \mathrm{~g} / \mathrm{L}(30 \mathrm{mM})$ at "cultivation day" five $(\mathrm{t}=0)$ and peak lipid occurred at $\mathrm{t}=6$ hours for strain PCH03 and t=12 hours for LB1H09 and PCH90. Lipid data for strain LB1H10 are not given since the change in the arrangement of cells evidenced by the FSC channel of the flow cytometer (Figure 2) confounds the interpretation of the FL1 channel signal. For strain LB1H10, 10.6\% (or $480 \mathrm{mg} / \mathrm{L}$ ) was consumed by $\mathrm{t}=12 \mathrm{~h}$ (Table 2). For this strain, there was no significant difference $(\mathrm{P}>0.05)$ between mixotrophic and photoautotrophic conditions in cellular lipid content over time (figure 5). This contrasts with the larger variation in growth rate and biomass production, of this strain, where cultures under 
mixotrophic conditions significantly outgrew ones under photoautotrophic conditions $(\mathrm{P}<0.05)$ (Figure 2). Increased biomass production brought about by xylose demonstrates the suitability of this strain for mixotrophic cultivation, and suggests that strain LB1H10 could potentially be useful for bioremediation of pulp \& paper industry wastes, especially for fractions where the hemicellulose that is present is unsuitable for chemical exploitation. This strain might also be of interest for the development of secondgeneration biofuels, where the efficient assimilation of xylose can represent a hurdle for some yeast strains [19].

Under mixotrophic conditions, most of the initial xylose remained unconsumed by cultures of strains PCH03 and PCH90 (Table 2). On average, only 195mg/L (PCH03) and $420 \mathrm{mg} / \mathrm{L}$ (PCH90) of xylose was consumed, representing a decrease of only $4.3 \%$ $(\mathrm{P}=0.02)$ and $9.3 \%(\mathrm{P}>0.05)$ of the initial sugar (Table 2$)$. The addition of xylose to cultures of the Trebouxiophyceae strains (LB1H09, $\mathrm{PCH} 03$ and $\mathrm{PCH} 90$ ) halted their growth, and although it induced lipid accumulation, the biomass productivity became significantly less than what was seen under photoautotrophic conditions (Figure 2). The photoautotrophic and mixotrophic lipid production of strain $\mathrm{PCH} 03$ were $37 \mathrm{mg} / \mathrm{L}$ and $69 \mathrm{mg} / \mathrm{L}$, while strain $\mathrm{PCH} 90$ gave $34 \mathrm{mg} / \mathrm{L}$ and $128 \mathrm{mg} / \mathrm{L}$, respectively (Table 2). The volumetric lipid production improvement of 1.86 and 3.76 fold was due to a substantial boost in cellular lipid content (2.7 and 4.1 fold respectively). Strain LB1H09 reached peak lipid content at 12 hours, at which point $12 \%(540 \mathrm{mg} / \mathrm{L})$ of the initial xylose had been consumed. Although this was almost three times the amount found for the other Trebouxiophyceae, the increase of cellular lipid content was within what was found for the others, from $16 \% \pm 1 \mathrm{dcw}$ to $47 \% \pm 8 \mathrm{dcw}$ on average for the three strains (Table 2). 
The increase in lipid productivity in the Trebouxiophyceae due to the increased cellular lipid content might not be directly associated with the direct conversion of xylose into this carbon reserve. The assimilation of this sugar requires its conversion into xylulose-5-phosphate, an intermediate of the pentose phosphate pathway [4]. This is a pathway with multiple reaction steps catalyzed by multiple enzymes, and it is likely that, even if constitutively expressed at a low basal level, appreciable xylose utilization by this pathway would require increased enzyme synthesis requiring a lengthy adaptation period.

Moreover, peak lipid accumulation was accompanied by an immediate growth halt, suggesting a disturbance of cell homeostasis. Interference in different metabolic pathways could cause this effect, and xylose has been shown to inactivate hexokinases and use hexose membrane transport systems in yeast $[20,21]$, or participate in the regulation of lipogenesis genes on mammals [28]. In fact, growth was re-established after 24 hours, where the end of this lag phase coincided with a decrease in cellular lipid content. This suggests that xylose addition might cause a direct interference with cellular metabolism or energy flux. Even though growth was reestablished after 24 hours, the cells still struggle to survive over the long term, losing substantial amounts of chlorophyll after two days (Figure 3), and decreasing in cell density.

These results suggest the potential use of xylose addition as a strategy for facile enhancement of algal lipid content in which an algal strain will either grow fast or accumulate large amounts of lipids in the presence of xylose. Three strains show a boost in lipid productivity (up to 3.8 fold), due to the increase in cellular lipid content (up to 4.1 fold) after exposure to xylose for a short period of time, 6 to 12 hours (Table 2),. This suggests that with these strains, xylose could be added as a lipid enhancer six to twelve 
hours before harvest. This would present certain advantages over the widely used practice of using nitrogen limitation to enhance lipid production, a process requiring relatively long incubation times (up to several days) and of limited efficiency [36]. The use of xylose to increase the lipid content could be employed in the form of an enhancement tank, located between the cultivation tanks and the centrifuges used for harvesting. This strategy would be suitable for cultivation in closed or open systems, with a retention time of only six to twelve hours, leading to an increase of more than three times the lipid productivity with just a fraction of the time required by the nitrogen starvation method. Moreover, as most of the xylose is still present in the solution after the lipid enhancement stage, it can be recycled into the enhancement tanks.

\section{Conclusions}

Productivity levels and production costs of algal biodiesel are still significant bottlenecks to the practical development of this biofuel. Xylose is an abundant molecule in nature, and an important constituent of many primary producers. Many microorganisms are naturally exposed to it, although in a very dilute form, in water bodies through the decomposition of plants.

Xylose also represents a cheap and readily available alternative carbon source, since it is the most abundant sugar in hemicellulose. Here the effects of xylose on growing algal cultures were analyzed using flow cytometry and three indigenous strains identified as Chlorella vulgaris and one from the Scenedesmaceae family. The addition of xylose produced a similar effect with the three Chlorella strains, inducing a fast 
accumulation of lipid, doubling or tripling the lipid content within 6 to 12 hours under mixotrophic conditions. These results suggest that xylose could be used in relatively small amounts as a lipid enhancer between the end of the photoautotrophic cultivation and the harvesting steps. After twenty-four hours exposition to xylose, the cells started losing chlorophyll and decreasing in size. The Scenedesmaceae strain was shown to be tolerant to the presence of xylose and to assimilate it under mixotrophic and heterotrophic growth conditions, boosting total biomass and lipid yields. Thus, this strain might be useful for biofuels production and for systems where xylose removal is a priority.

\section{Acknowledgments}

This research was supported by grants from FQRNT (Le Fonds Québécois de la recherche sur la nature et les technologies), programme de recherche en partenariat contribuant à la séquestration des gaz à effet de serre (2011-GZ-141307) P.C.H., by Biorémédiation d'effluents industriel provenant de l'industrie des biocarburants via la culture de biomasse algale mixotrophe (Numéro de référence : 24198) and by CRSNGSTPGP 447266-13 Bio-oil Recovery \& CO2 Recycling by Waste Stream Enhanced Microalgal Growth \& Low Energy CO2-Related Extraction. PCH holds a National Research Council Senior Research Associateship Award at the Life Sciences Research Center, Department of Biology, United States Air Force Academy. The views expressed in this article are those of the authors and do not reflect the official policy or position of the United States Air Force, the Department of Defense, or the US Government. We thank Patrick McGinn and Shabana Bhatti (Halifax, NS), NRC Institute for Marine Biosciences, for advice and training at the Ketch Harbour Research Center. 


\section{References}

[1] A.E.M. Abdelaziz, G.B. Leite, P.C. Hallenbeck, Addressing the challenges for sustainable production of algal biofuels: I. Algal strains and nutrient supply, Environmental Technology. 34 (2013) 1783-1805. doi:10.1080/09593330.2013.827748.

[2] G.B. Leite, A.E.M. Abdelaziz, P.C. Hallenbeck, Algal biofuels: Challenges and opportunities, Bioresour Technol. (2013) 1-8.

doi:10.1016/j.biortech.2013.02.007.

[3] M.E. Huntley, Z.I. Johnson, S.L. Brown, D.L. Sills, L. Gerber, I. Archibald, et al., Demonstrated large-scale production of marine microalgae for fuels and feed, Algal. 10 (2015) 249-265. doi:10.1016/j.algal.2015.04.016.

[4] C. Zhang, Y. Zhang, B. Zhuang, X. Zhou, Strategic enhancement of algal biomass, nutrient uptake and lipid through statistical optimization of nutrient supplementation in coupling Scenedesmus obliquus-like microalgae cultivation and municipal wastewater treatment, Bioresour Technol. 171 (2014) 71-79. doi:10.1016/j.biortech.2014.07.060.

[5] D.L. Sutherland, C. Howard-Williams, M.H. Turnbull, P.A. Broady, R.J. Craggs, Enhancing microalgal photosynthesis and productivity in wastewater treatment high rate algal ponds for biofuel production, Bioresour Technol. 184 (2015) 222229. doi:10.1016/j.biortech.2014.10.074.

[6] G. Chen, L. Zhao, Y. Qi, Enhancing the productivity of microalgae cultivated in wastewater toward biofuel production: A critical review, Appl Energy. 137 (2015) 282-291. doi:10.1016/j.apenergy.2014.10.032.

[7] P.J. McGinn, K.E. Dickinson, S. Bhatti, J.-C. Frigon, S.R. Guiot, S.J.B. O'Leary, Integration of microalgae cultivation with industrial waste remediation for biofuel and bioenergy production: opportunities and limitations, Photosynth Res. 109 (2011) 231-247. doi:10.1007/s11120-011-9638-0.

[8] A.E.M. Abdelaziz, G.B. Leite, M.A. Belhaj, P.C. Hallenbeck, Screening microalgae native to Quebec for wastewater treatment and biodiesel production, Bioresour Technol. (2014) 1-33. doi:10.1016/j.biortech.2014.01.114.

[9] A. Bhatnagar, S. Chinnasamy, M. Singh, K.C. Das, Renewable biomass production by mixotrophic algae in the presence of various carbon sources and wastewaters, Appl Energy. 88 (2011) 3425-3431.

doi:10.1016/j.apenergy.2010.12.064.

[10] V. Turon, E. Trably, E. Fouilland, J.P. Steyer, Growth of Chlorella sorokiniana on a mixture of volatile fatty acids: The effects of light and temperature, Bioresour Technol. 198 (2015) 852-860. doi:10.1016/j.biortech.2015.10.001.

[11] K. Paranjape, G.B. Leite, P.C. Hallenbeck, Strain variation in microalgal lipid production during mixotrophic growth with glycerol, Bioresour Technol. 204 (2016) 80-88. doi:10.1016/j.biortech.2015.12.071.

[12] Y. Zheng, X. Yu, T. Li, X. Xiong, S. Chen, Induction of D-xylose uptake and expression of $\mathrm{NAD}(\mathrm{P}) \mathrm{H}$-linked xylose reductase and NADP + -linked xylitol dehydrogenase in the oleaginous microalga Chlorella sorokiniana, Biotechnol Biofuels. 7 (2014) 125. doi:10.1186/s13068-014-0125-7.

[13] S. Yang, G. Liu, Y. Meng, P. Wang, S. Zhou, H. Shang, Utilization of xylose as 
a carbon source for mixotrophic growth of Scenedesmus obliquus, Bioresour Technol. 172 (2014) 180-185. doi:10.1016/j.biortech.2014.08.122.

[14] G.B. Leite, K. Paranjape, A.E.M. Abdelaziz, P.C. Hallenbeck, Utilization of biodiesel-derived glycerol or xylose for increased growth and lipid production by indigenous microalgae, Bioresour Technol. 184 (2015) 123-130. doi:10.1016/j.biortech.2014.10.117.

[15] Z. Xie, W. Lin, J. Luo, Promotion of microalgal growth by co-culturing with Cellvibrio pealriver using xylan as feedstock, (2015) 1-5. doi:10.1016/j.biortech.2015.10.058.

[16] H.V. Scheller, P. Ulvskov, Hemicelluloses, Annu. Rev. Plant Biol. 61 (2010) 263-289. doi:10.1146/annurev-arplant-042809-112315.

[17] P. Moniz, A.L. Ho, L.C. Duarte, S. Kolida, R.A. Rastall, H. Pereira, et al., Assessment of the bifidogenic effect of substituted xylo-oligosaccharides obtained from corn straw, Carbohydrate Polymers. 136 (2016) 466-473. doi:10.1016/j.carbpol.2015.09.046.

[18] V. Novy, K. Longus, B. Nidetzky, From wheat straw to bioethanol: integrative analysis of a separate hydrolysis and co-fermentation process with implemented enzyme production, Biotechnol Biofuels. 8 (2015) 46. doi:10.1186/s13068-0150232-0.

[19] X. Li, V.Y. Yu, Y. Lin, K. Chomvong, R. Estrela, A. Park, et al., Expanding xylose metabolism in yeast for plant cell wall conversion to biofuels, Elife. 4 (2015) e05896. doi:10.7554/eLife.05896.

[20] R. Fernández, P. Herrero, F. Moreno, Inhibition and inactivation of glucosephosphorylating enzymes from Saccharomyces cerevisiae by D-xylose, J. Gen. Microbiol. 131 (1985) 2705-2709. doi:10.1099/00221287-131-10-2705.

[21] W.-J. Lee, M.-D. Kim, Y.-W. Ryu, L.F. Bisson, J.-H. Seo, Kinetic studies on glucose and xylose transport in Saccharomyces cerevisiae, Appl Microbiol Biotechnol. 60 (2002) 186-191. doi:10.1007/s00253-002-1085-6.

[22] P.C. Hallenbeck, G.B. Leite, A.E.M. Abdelaziz, Exploring the diversity of microalgal physiology for applications in wastewater treatment and biofuel production, Algal Research. 6 (2014) 111-118. doi:10.1016/j.algal.2014.09.006.

[23] R.A. Andersen, Algal Culturing Techniques, Academic Press, 2005.

[24] T. Govender, L. Ramanna, I. Rawat, F. Bux, BODIPY staining, an alternative to the Nile Red fluorescence method for the evaluation of intracellular lipids in microalgae, Bioresour Technol. 114 (2012) 507-511.

doi:10.1016/j.biortech.2012.03.024.

[25] L. Brennan, A. Blanco Fernández, A.S. Mostaert, P. Owende, Enhancement of BODIPY505/515 lipid fluorescence method for applications in biofuel-directed microalgae production, Journal of Microbiological Methods. 90 (2012) 137-143. doi:10.1016/j.mimet.2012.03.020.

[26] E. Bertozzini, L. Galluzzi, A. Penna, M. Magnani, Application of the standard addition method for the absolute quantification of neutral lipids in microalgae using Nile red, Journal of Microbiological Methods. 87 (2011) 17-23. doi:10.1016/j.mimet.2011.06.018.

[27] W. Chen, C. Zhang, L. Song, M. Sommerfeld, Q. Hu, A high throughput Nile red method for quantitative measurement of neutral lipids in microalgae, Journal of 
Microbiological Methods. 77 (2009) 41-47. doi:10.1016/j.mimet.2009.01.001.

[28] D. Elsey, D. Jameson, B. Raleigh, M.J. Cooney, Fluorescent measurement of microalgal neutral lipids, Journal of Microbiological Methods. 68 (2007) 639642. doi:10.1016/j.mimet.2006.11.008.

[29] G.L. Miller, Use of Dinitrosalicylic Acid Reagent for Determination of Reducing Sugar, Anal. Chem. 31 (1959) 426-428. doi:10.1021/ac60147a030.

[30] M. Abo-Hashesh, Microaerobic dark fermentative hydrogen production by the photosynthetic bacterium, Rhodobacter capsulatus JP91, International Journal of Low Carbon Technologies (2012). doi:10.1093/ijlct/cts011.

[31] E.F. López, E.F. Gómez, Simultaneous determination of the major organic acids, sugars, glycerol, and ethanol by HPLC in grape musts and white wines, Journal of Chromatographic .... (1996).

[32] S.J. Edmundson, M.H. Huesemann, The dark side of algae cultivation: Characterizing night biomass loss in three photosynthetic algae, Chlorella sorokiniana, Nannochloropsis salina and Picochlorum sp, Algal. 12 (2015) 470476. doi:10.1016/j.algal.2015.10.012.

[33] M.S. Cooper, W.R. Hardin, T.W. Petersen, R.A. Cattolico, Visualizing "green oil" in live algal cells, Journal of Bioscience and Bioengineering. 109 (2010) 198-201. doi:10.1016/j.jbiosc.2009.08.004.

[34] T. Mutanda, D. Ramesh, S. Karthikeyan, S. Kumari, A. Anandraj, F. Bux, Bioprospecting for hyper-lipid producing microalgal strains for sustainable biofuel production, Bioresour Technol. 102 (2011) 57-70.

doi:10.1016/j.biortech.2010.06.077.

[35] K. Iizuka, Y. Horikawa, ChREBP: a glucose-activated transcription factor involved in the development of metabolic syndrome, Endocrine Journal. (2008). doi:10.1507/endocrj.K07E-110.

[36] S. Zhu, Y. Wang, J. Xu, C. Shang, Z. Wang, J. Xu, et al., Luxury uptake of phosphorus changes the accumulation of starch and lipid in Chlorella sp. under nitrogen depletion, Bioresour Technol. 198 (2015) 165-171. doi:10.1016/j.biortech.2015.08.142. 


\section{Tables}

Table 1: Growth (And Loss) Rates During the First 24hrs After Addition Of Xylose

Growth rate $(\mu)$ measured between $t=0$ (addition of xylose) and $t=24 \mathrm{hrs}$.

Abbreviations: $-\mathrm{xyl}=$ no $x y l o s e$ added $;+x y l=$ xylose added .

\begin{tabular}{|c|c|c|c|c|}
\hline \multirow{3}{*}{ Strains } & \multicolumn{4}{|c|}{$\mu\left(\right.$ day $\left.^{-1}\right)$} \\
\hline & \multicolumn{2}{|c|}{ Light } & \multicolumn{2}{|c|}{ Dark } \\
\hline & $-x y l$ & $+x y l$ & $-x y l$ & $+x y l$ \\
\hline LB1H09 & $\begin{array}{c}0.215 \\
( \pm 0.065)\end{array}$ & $\begin{array}{c}-0.005 \\
( \pm 0.061)\end{array}$ & $\begin{array}{c}-0.725 \\
( \pm 0.053)\end{array}$ & $\begin{array}{c}-0.550 \\
( \pm 0.158)\end{array}$ \\
\hline LB1H10 & $\begin{array}{c}0.415 \\
( \pm 0.048)\end{array}$ & $\begin{array}{c}0.272 \\
( \pm 0.100)\end{array}$ & $\begin{array}{c}-0.112 \\
( \pm 0.047)\end{array}$ & $\begin{array}{c}0.021 \\
( \pm 0.229)\end{array}$ \\
\hline РCH03 & $\begin{array}{c}0.290 \\
( \pm 0.042)\end{array}$ & $\begin{array}{c}0.075 \\
( \pm 0.075)\end{array}$ & $\begin{array}{c}-1.051 \\
( \pm 0.191)\end{array}$ & $\begin{array}{c}-0.371 \\
( \pm 0.119)\end{array}$ \\
\hline PCH90 & $\begin{array}{c}0.361 \\
( \pm 0.009)\end{array}$ & $\begin{array}{c}0.129 \\
( \pm 0.046)\end{array}$ & $\begin{array}{c}-1.034 \\
( \pm 0.108)\end{array}$ & $\begin{array}{c}-0.312 \\
( \pm 0.085)\end{array}$ \\
\hline
\end{tabular}


Table 2 : Effect of Xylose on Lipid and Biomass Productivity under

\section{Photoautotrophic and Mixotrophic Conditions}

Maximum lipid and biomass production measured at peak lipid content as seen in figure

5. 


\begin{tabular}{|c|c|c|c|c|c|c|c|c|c|c|}
\hline Strain & $\begin{array}{l}\text { Cultiv. } \\
\text { Days }\end{array}$ & Peak & $\begin{array}{c}\text { Biomass } \\
\text { mg/L }\end{array}$ & $\begin{array}{l}\text { Biomass } \\
\text { mg/L/day }\end{array}$ & $\begin{array}{c}\text { Xylose } \\
\text { Depletion } \\
\text { mg/L }\end{array}$ & $\begin{array}{l}\text { Lipid } \\
\text { mg/L }\end{array}$ & $\begin{array}{c}\text { Lipid } \\
\text { mg/L/day }\end{array}$ & $\begin{array}{l}\text { Lipid Prod. } \\
\text { Increase } \\
\text { (Fold) }\end{array}$ & $\begin{array}{c}\text { Lipid Content } \\
\text { \% DW }\end{array}$ & $\begin{array}{c}\text { Lipid Cont. } \\
\text { Increase } \\
\text { (Fold) }\end{array}$ \\
\hline LB1H09 & 5.5 & $\mathrm{t}=12 \mathrm{~h}$ & $332 \pm 23$ & $60.4 \pm 4.2$ & ---- & $54 \pm 6$ & $9.8 \pm 1.1$ & ----- & $16 \% \pm 1$ & ----- \\
\hline LB1H09 + Xylose & 5.5 & $\mathrm{t}=12 \mathrm{~h}$ & $195 \pm 34$ & $35.5 \pm 6.2$ & $540 \pm 90$ & $94 \pm 33$ & $17.1 \pm 6$ & $1.7 \mathrm{x}$ & $47 \% \pm 8$ & $2.9 \mathrm{x}$ \\
\hline LB1H10 & 5.5 & $t=12 h$ & $187 \pm 24$ & $34.1 \pm 4.2$ & ---- & ------ & ----- & ----- & ------ & ----- \\
\hline LB1H10 + Xylose & 5.5 & $\mathrm{t}=12 \mathrm{~h}$ & $191 \pm 29$ & $34.7 \pm 5.2$ & $480 \pm 150$ & ----- & ------ & ----- & ------ & ----- \\
\hline PCH03 & 5.25 & $t=6 h$ & $262 \pm 31$ & $49.9 \pm 5.9$ & ---- & $37 \pm 8$ & $7 \pm 1.5$ & ----- & $15 \% \pm 3$ & ------ \\
\hline PCH03 + Xylose & 5.25 & $t=6 h$ & $182 \pm 42$ & $34.7 \pm 8$ & $195 \pm 165$ & $69 \pm 19$ & $13.1 \pm 3.6$ & $1.9 \mathrm{x}$ & $41 \% \pm 6$ & $2.7 \mathrm{x}$ \\
\hline PCH90 & 5.5 & $\mathrm{t}=12 \mathrm{~h}$ & $220 \pm 23$ & $39.9 \pm 4.2$ & ---- & $34 \pm 8$ & $6.2 \pm 1.5$ & ----- & $15 \% \pm 2$ & ------ \\
\hline PCH90 + Xylose & 5.5 & $\mathrm{t}=12 \mathrm{~h}$ & $208 \pm 40$ & $37.8 \pm 7.3$ & $420 \pm 60$ & $128 \pm 34$ & $23.3 \pm 6.2$ & $3.8 \mathrm{x}$ & $61 \% \pm 5$ & $4.1 \mathrm{x}$ \\
\hline
\end{tabular}

$(---)=$ not applicable. 


\section{Figures}

\section{Figure 1: Phylogenetic Tree}

Neighbor joining phylogenetic tree based on the alignment of the internal region of the 18S rDNA containing 912bp. The strain LB1H10 was placed within the class Chlorophyceae while the others strains were classified as different strains of Chlorella vulgaris (class Trebouxiophyceae). 18S rDNA (912bp) was amplified, sequenced, compared against the NCBI database, aligned and compared to other known species for their placement in the proper phylogenetic clade. The tree is drawn to scale, with branch lengths in the same units as those of the evolutionary distances used to infer the phylogenetic tree.

\section{Figure 2: $\mathrm{OD}_{630}$ and FSC as a function of Time}

Optical density (left) and mean Forward Scatter (FSC - right) were recorded over time. Optical density is correlated with the bulk biomass, while the FSC channel is related to average cell size. The vertical dotted line represents time $=0$ when the first stage cultures were split into four and xylose was added. Circles represent the group with xylose (mixotrophic and heterotrophic) and the triangles represent the groups that did not receive xylose (photoautotrophic and dark autotrophic). Clear symbols are the cultures incubated in the light (photoautotrophic and mixotrophic) and the filled symbols are the cultures incubated in the dark (heterotrophic and dark autotrophic).

\section{Figure 3: Visual Color Changes with Time}


Photographs of the Erlenmeyer flasks at different time points: 48, 72 and 96 hours after xylose addition ( $\mathrm{t}=0$ ). Strains are, from left to right: LB1H09 (Chlorella $\mathrm{sp}$ ); LB1H10 (Scenedesmaceae); PCH03 (Chlorella sp.) and PCH90 (Chlorella sp.); where the column on the left presents the mixotrophic cultures (light + xylose), and the right column the photoautotrophic replicates. All the strains from the genus Chlorella showed continuous bleaching after exposure to xylose while the member of the Scenedesmaceae family (LB1H10) had the same visual appearance for both mixotrophic and photoautotrophic cultures throughout the experiment..

\section{Figure 4: pH changes during cultivation}

The $\mathrm{pH}$ of the medium $\mathrm{pH}$ of the photoautotrophic (triangles) and mixotrophic cultures (circles) at different time points. The $\mathrm{pH}$ was measured prior the inoculum and daily after the addition of xylose $(\mathrm{t}=0)$.

\section{Figure 5: Evolution of Lipid and Chlorophyll Content over Time}

Flow cytometric mean fluorescence measurements of channels FL1 (lipids) and FL3 (chlorophyll) are shown as a function of time. The vertical dotted line represents the moment when xylose was added to the treated groups (time $=0$ ). For three of the strains, a fast accumulation of lipids can be seen over the first twelve hours after the addition of xylose, followed by a continuous decrease in chlorophyll content. Circles represent the treated group (xylose added) and the triangles represent the untreated groups (received no xylose) $($ clear=light; black=dark) 

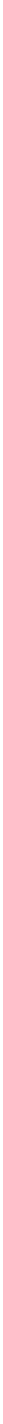

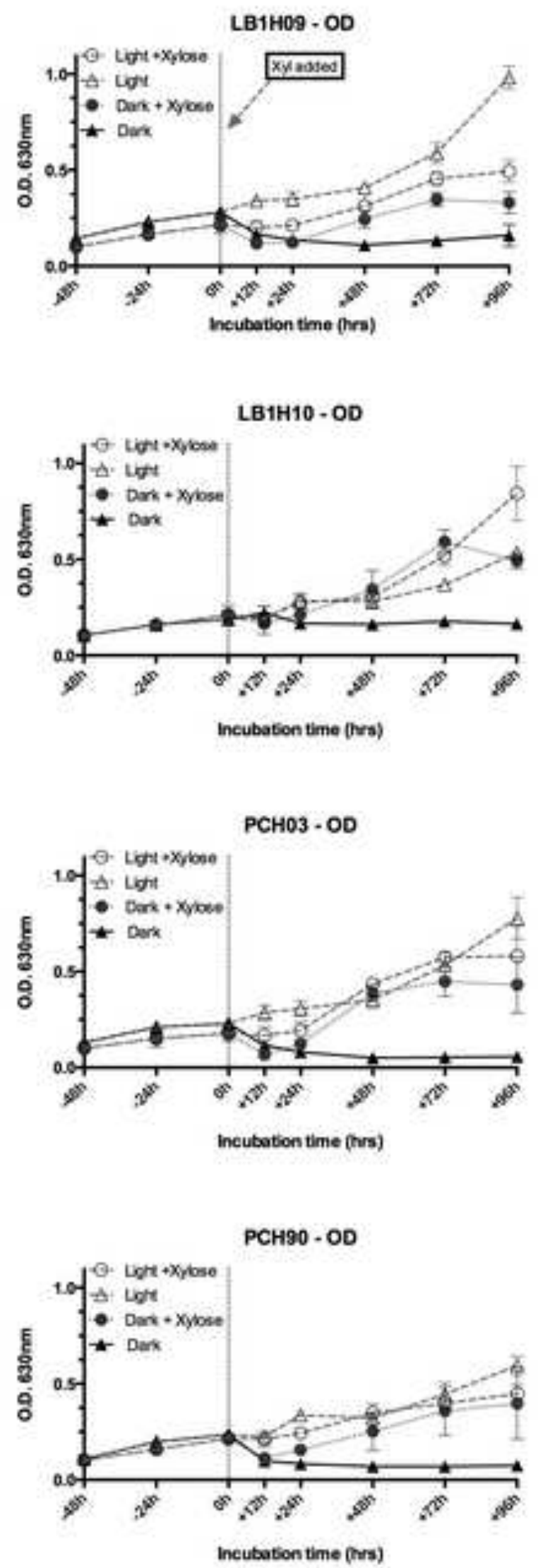
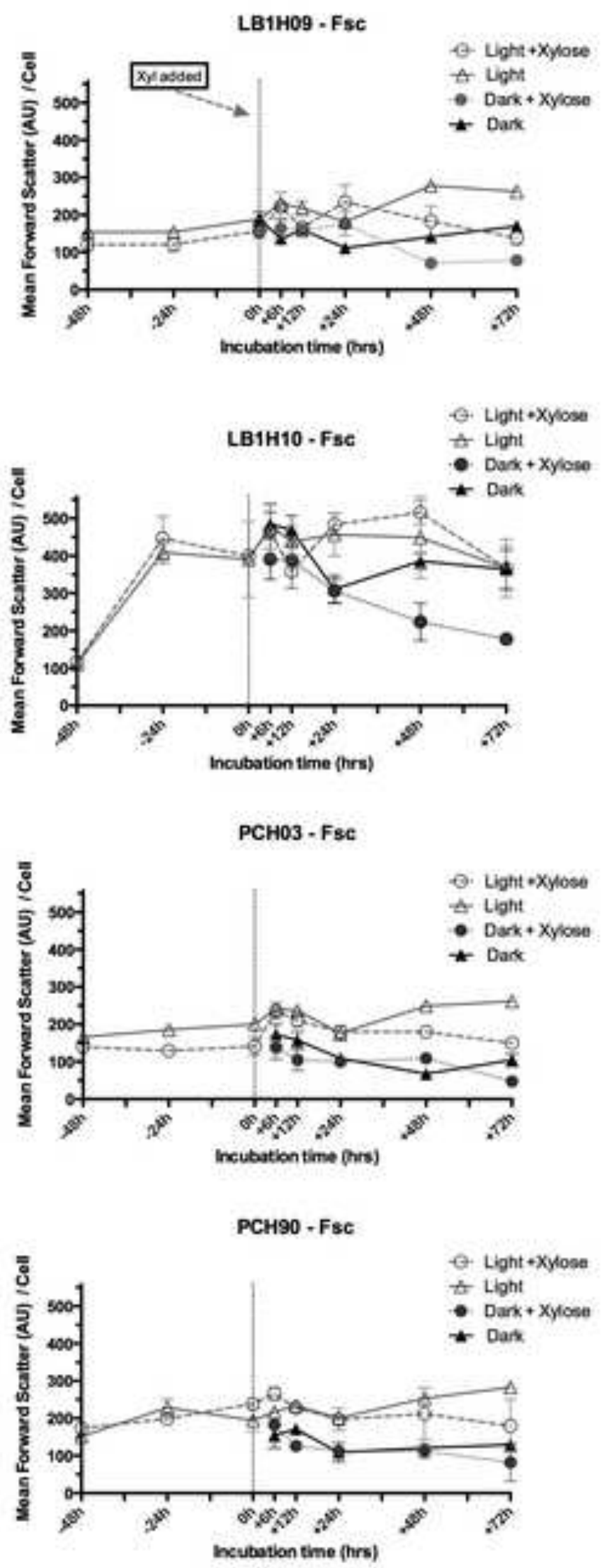
Cultures pictures:

26-sept-2014@13:00 (T=48hrs)

Light + Xylose

Light
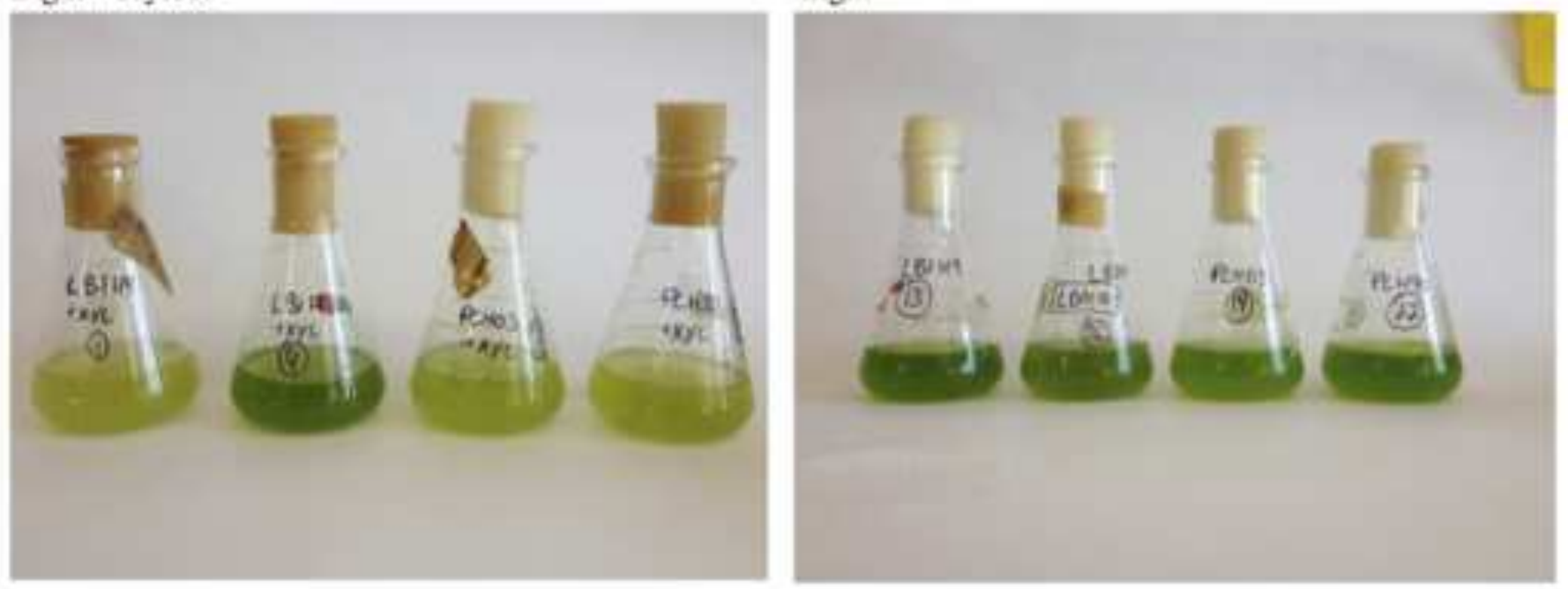

27-sept-2014@11:10 (t=72hrs)

Light + Xylose

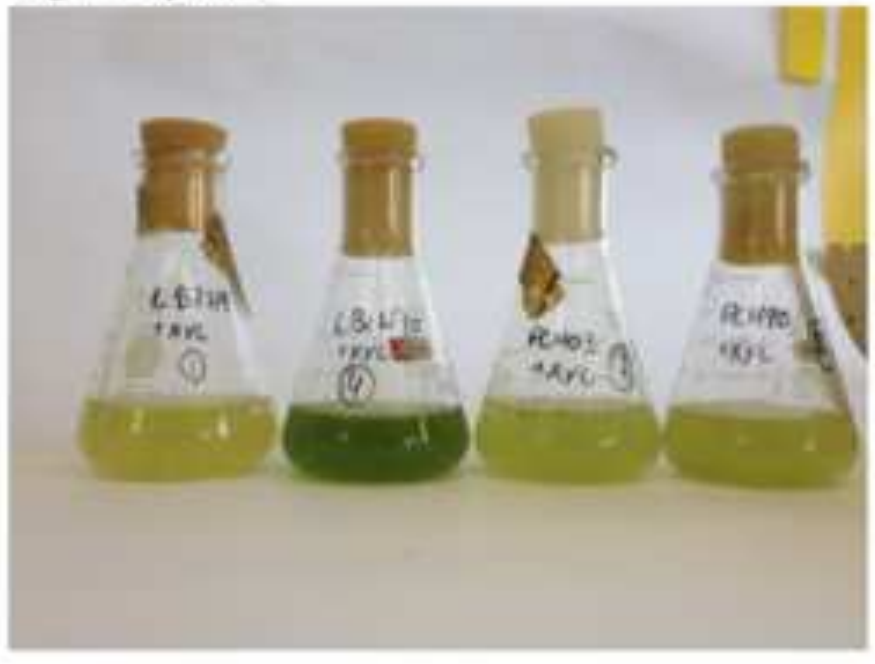

Light

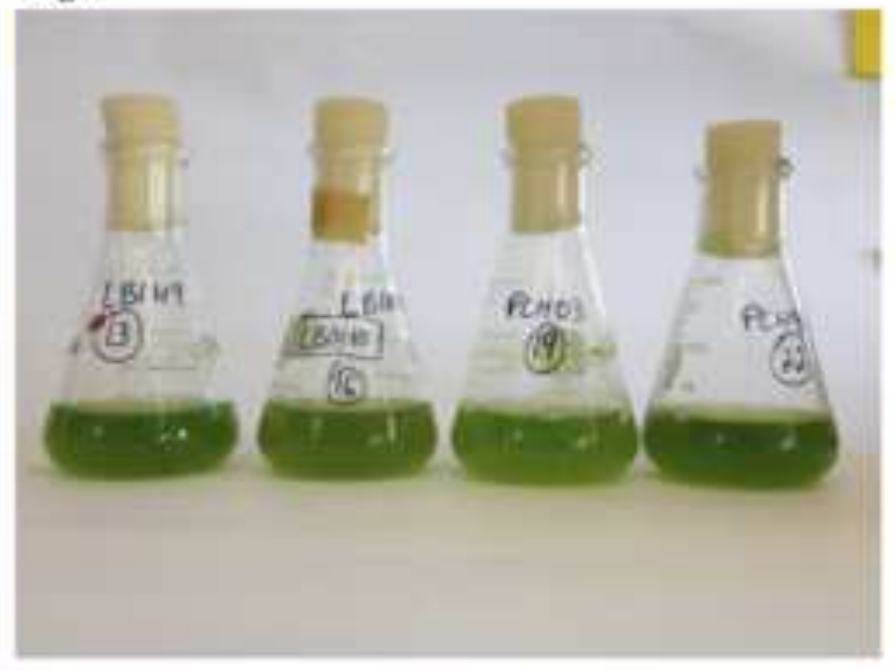

\section{8 sept 2014 (t=96hrs)}

Light + Xylose

Light
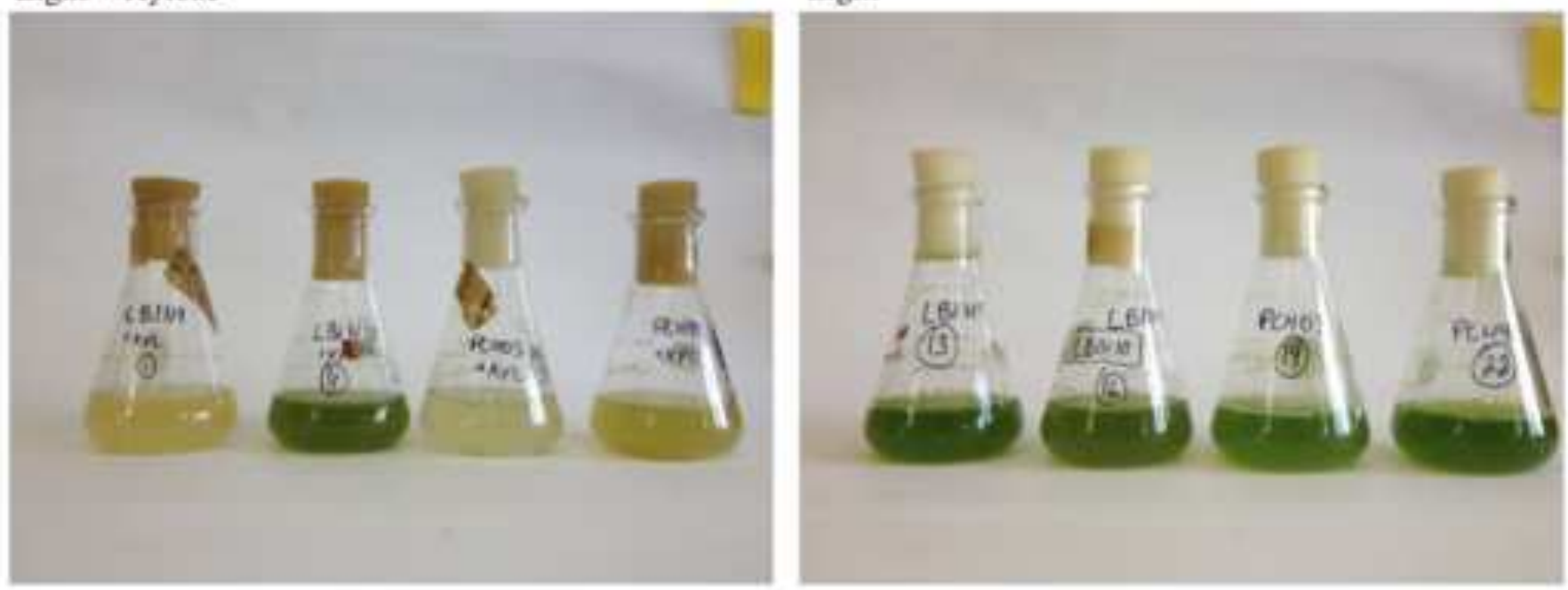

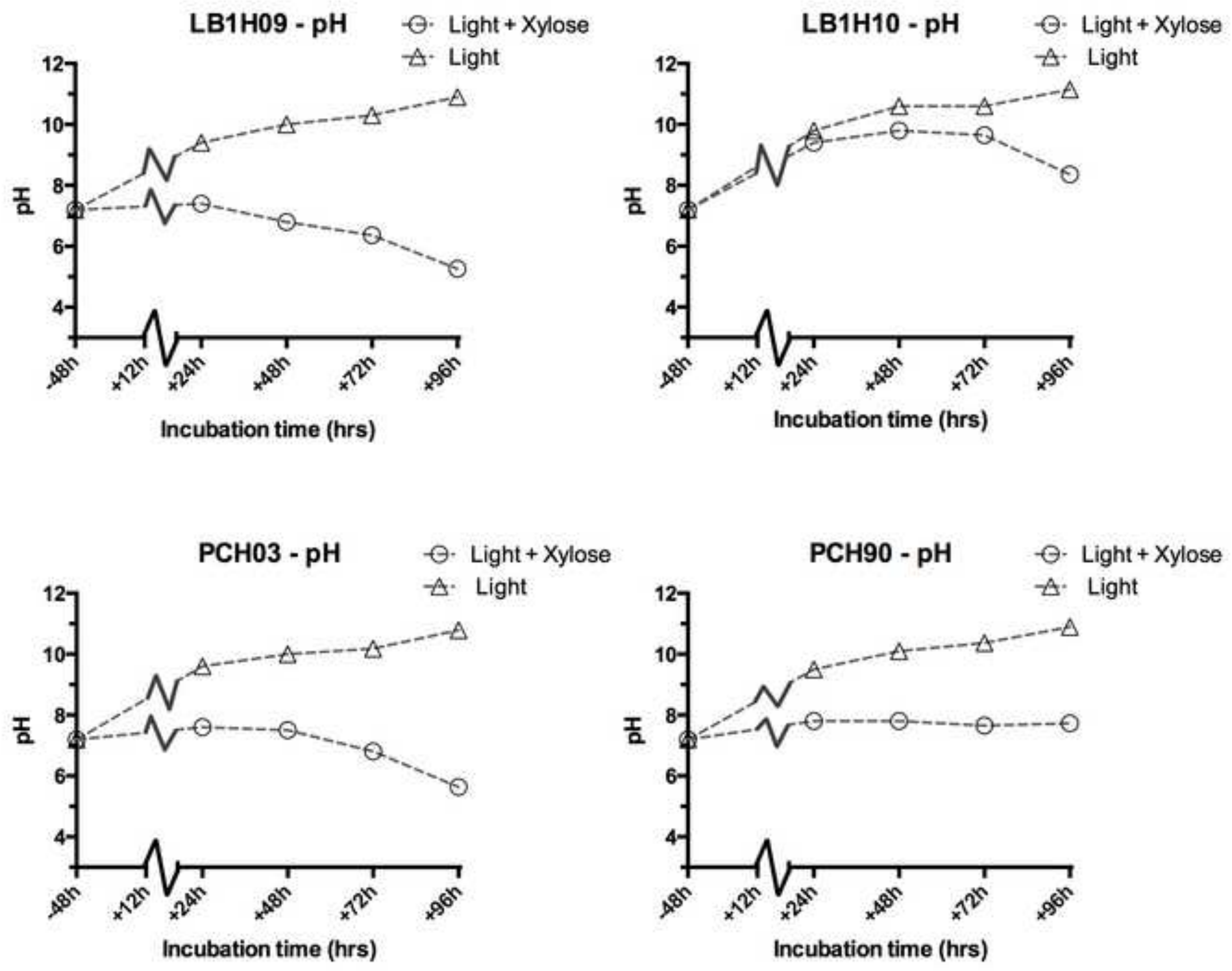
LB1HO9 - FL1

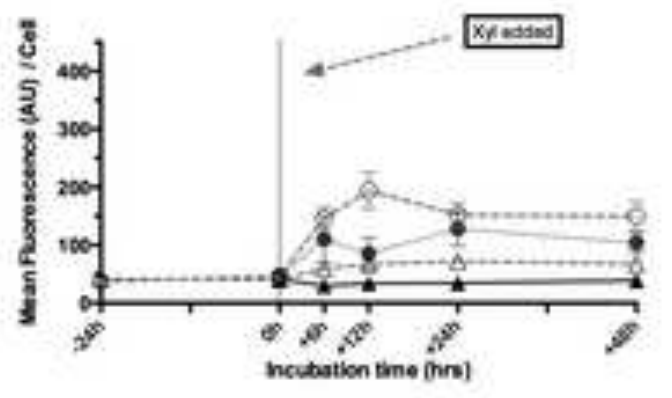

LB1H10 - FL1

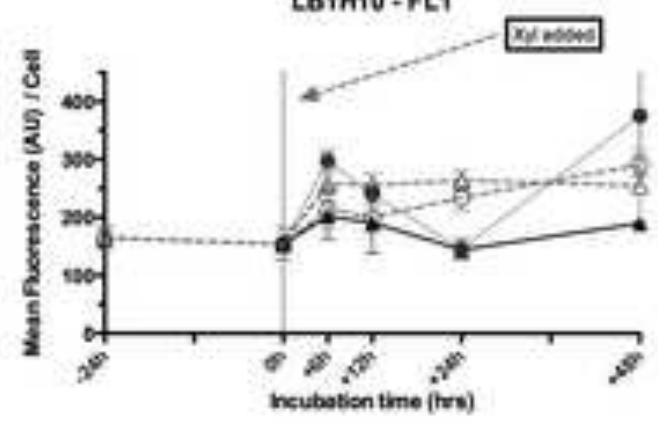

PCHOS - FLI

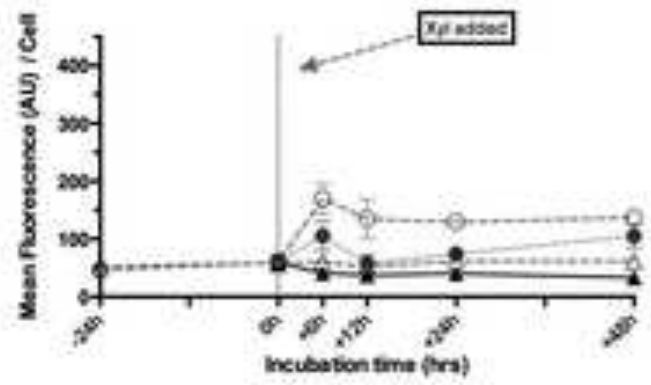

PCkso - FL1

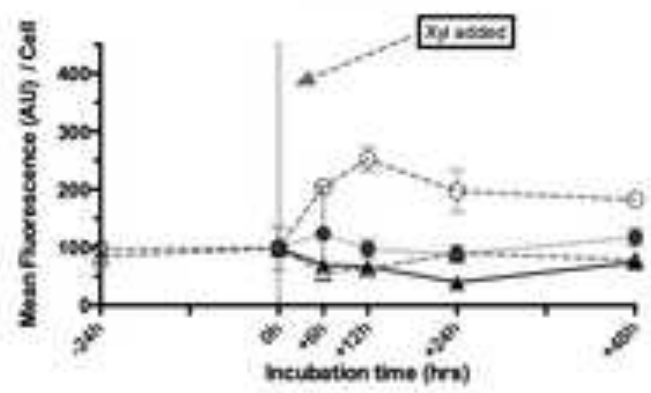

- L. Light -xylcae

A. Lent

- Dark + xyose

* Das

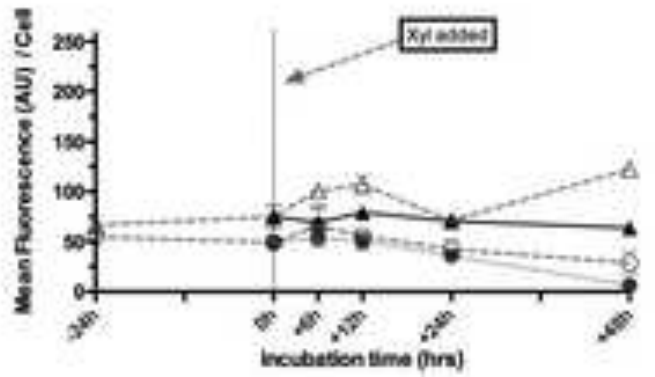

9. Light +X Xybese

d. topt

- Dark-Xytos

* Das.
Q. Light + Xylose

te Light

- Dax + Xyole

+ Dosk

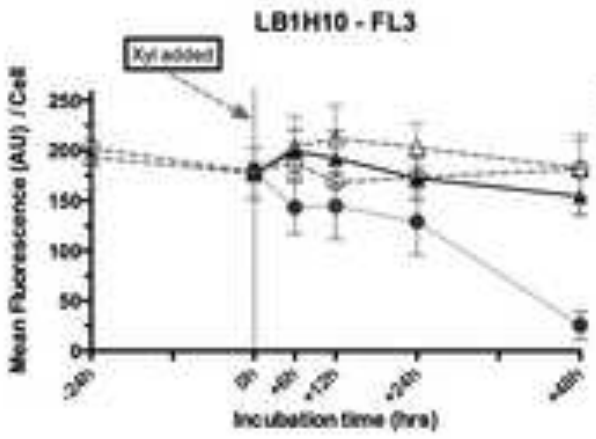

7- Loght +Xyoue

A. Light

- Dark + Xylose

$\star 0$ oas

9. Ueht +xyose

is. Uipar

- Dent + Xyloses

tr Dark.

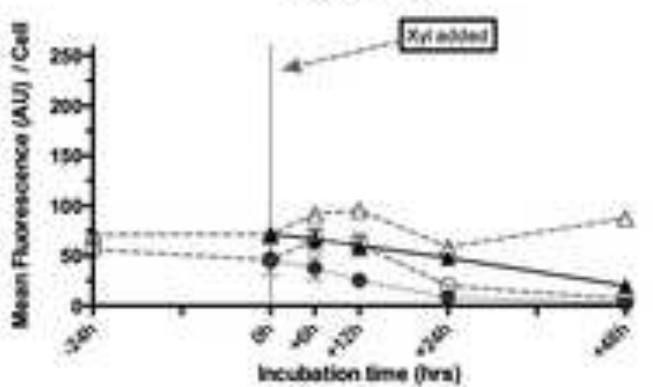

PCI490 - FL3

6. Light + Xylose

4. Lipht

- Dak + Xyloas

- Dak

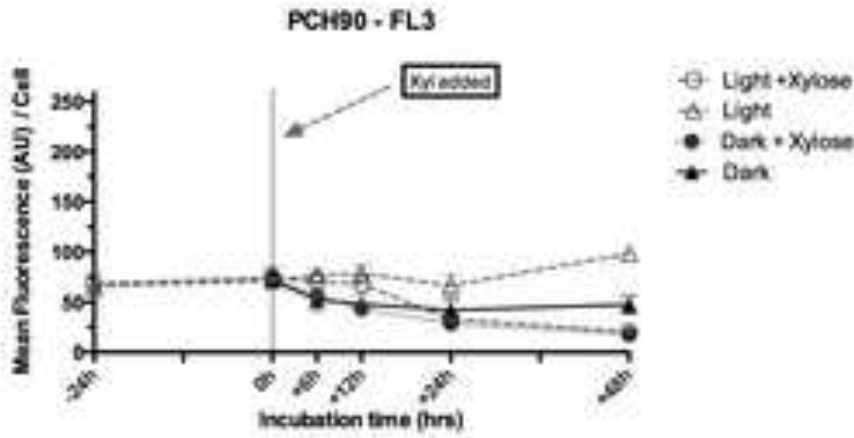

Q. Loht +xyose

A. Light

- Dark+Xyole

$\downarrow$ Dax. 